\title{
EFECTOS PECUNIARIOS Y NO PECUNIARIOS DEL DESAJUSTE ENTRE EMPLEO Y ÁREA DE ESTUDIO: EVIDENCIA A PARTIR DE LA IMPLEMENTACIÓN DE UN SISTEMA TRANSVERSAL DE SEGUIMIENTO DE TITULADOS ${ }^{1}$
}

\author{
Andrés Muñoz ${ }^{2}$ \\ Sebastián Guinguis ${ }^{3}$ \\ Alejandra Reyes ${ }^{4}$ \\ José Ferreiro ${ }^{5}$
}

RESUMEN

El desajuste entre empleo y área de estudio ha recibido especial atención en la literatura (Robst, 2007). Las investigaciones muestran que los trabajadores empleados en áreas no relacionadas con sus carreras obtienen salarios menores que aquellos con el mismo nivel de escolaridad empleados en puestos pertinentes. Este trabajo estima el efecto salarial del desajuste educacional y analiza sus implicancias sobre la satisfacción con el empleo. Para ello, analiza datos de una encuesta de titulados de una universidad estatal metropolitana en el período 2014-2017. El artículo busca responder a las siguientes preguntas: ¿Cómo afecta el empleo fuera del área de estudio a los salarios y a la satisfacción laboral? ¿Qué características de las trayectorias educativas y laborales explican el desajuste entre área de estudio y tipo de empleo? Este estudio muestra que quienes se emplean en puestos no relacionados con su área de estudio obtienen salarios menores que los que reportan tener un empleo que sí se relaciona con esta. Asimismo, el empleo no relacionado con la carrera afecta el grado de satisfacción, especialmente en el caso de los hombres a tres años después de haber egresado, dadas las menores expectativas de desarrollo profesional en el lugar de trabajo actual.

Conceptos clave: retornos a la educación superior, desajuste educacional, economía de la educación, pertinencia del empleo.

ECONOMIC AND NON-ECONOMIC EFFECTS OF THE MISMATCH BETWEEN ONE'S JOB AND FIELD OF STUDY: EVIDENCE FROM THE IMPLEMENTATION OF AN INSTITUTIONAL FOLLOW-UP SURVEY OF GRADUATES

ABSTRACT

Misalignment between a worker's field of study and job has received much attention in the literature (Robst, 2007). Research shows that workers employed in areas unrelated to their fields of study earn lower salaries than those with the same level of education yet employed in positions related to their

Proyecto apoyado financieramente por CNED/Convocatoria 2017. Los autores agradecen la valiosa colaboración de José Cáceres, Diego Altamirano, María José Vásquez, Marcela Ortega y Cristian Olate, en el trabajo de construcción y análisis de bases de datos y la revisión de versiones previas del documento.

2 Universidad Tecnológica Metropolitana, Santiago, Chile. Contacto: amunozo@utem.cl

3 Universidad Tecnológica Metropolitana, Santiago, Chile. Contacto: sguinguis@utem.cl

4 Universidad Tecnológica Metropolitana, Santiago, Chile. Contacto: areyes@utem.cl

5 Merlin Research, Santiago, Chile. Contacto: josemanuelferreiro@yahoo.es 
education. This paper estimates the educational mismatch's effect on wages and analyses its implications on job satisfaction, through analyzing data gleamed from a survey of a public metropolitan university's graduates, applied over the period of 2014-2017. The study seeks to answer the following: How does working outside of one's degree field affect earnings and job satisfaction? Which characteristics of educational and work pathways explain the misalignment between the field of study and type of employment? This study shows that those who are employed in positions unrelated to their areas of study earn less and enjoy less job satisfaction than those whose work is aligned with their field of study. Likewise, effects on job satisfaction are more pronounced three years after graduation, particularly for men with business and administration degrees compared to technology graduates, given lower expectations for professional development in their current positions.

Key concepts: returns on higher education, educational mismatch, economics of education; employment relevance.

\section{Introducción}

La instalación del sistema de aseguramiento de la calidad de la educación superior en Chile ha impulsado estrategias institucionales de seguimiento de trayectorias laborales de egresados del sistema de educación superior. Mientras la institucionalidad pública informa estándares de participación laboral e ingresos del trabajo al primer y cuarto año de titulación (Ministerio de Educación de Chile, Mineduc, 2018), los estudios nacionales se centran en el análisis de los retornos salariales de los egresados de la educación superior. La evidencia en esta línea sugiere la existencia de retornos positivos, pero heterogéneos según el tipo de institución o carrera, usando como línea de base los salarios de egresados de educación secundaria (Meller, 2010; Sotomayor, 2015; Urzúa, 2012). Otras fuentes de heterogeneidad en los retornos salariales, como las discutidas en las áreas de sobreeducación y desajuste educacional (Hartog, 2000; Robst, 2007), han quedado desatendidas en los estudios nacionales en torno a las trayectorias laborales de egresados de educación superior.

La literatura en el área de sobreeducación analiza el efecto de las diferencias entre el nivel de escolaridad alcanzado por un trabajador y el requerido en su lugar de trabajo en cuanto a salarios, satisfacción laboral, niveles de rotación y productividad (Hartog, 2000). Bajo este enfoque, los trabajadores que poseen mayor o menor escolaridad que lo solicitado por sus puestos de trabajo se denominan sobre o subeducados, según corresponda. La 
investigación en esta área concluye que la variabilidad en los retornos salariales entre trabajadores con igual escolaridad se explica por los distintos niveles de escolaridad requeridos en los puestos de trabajo. El retorno salarial óptimo se observa en egresados cuya escolaridad se ajusta completamente a la demandada en el empleo, siendo el retorno salarial de aquellos sobreeducados menor al óptimo e incluso negativo en el caso de los trabajadores subeducados (Hartog, 2000).

Un segundo enfoque en la línea del desajuste educacional analiza el impacto del desajuste entre el área de estudio y el tipo de empleo sobre los salarios de los egresados. Usando datos de una encuesta nacional de egresados en Estados Unidos, Robst (2007) consignó que 45\% de ellos reportó que su trabajo estaba parcial o completamente desajustado con su área de estudio. Sus resultados indican que la prevalencia del desajuste es mayor entre los egresados de áreas de estudio orientadas a la formación de competencias genéricas, comparado con egresados de áreas de estudio orientadas a la formación de competencias específicas. En contraposición, los egresados de áreas de estudio orientadas a la formación de competencias específicas enfrentan un mayor costo salarial asociado al desajuste entre tipo de empleo y área de estudio, comparado con los egresados de áreas de estudio orientadas a la formación de competencias genéricas. Estos resultados indican una relación inversa entre la probabilidad y el costo del desajuste entre áreas del conocimiento orientadas a formaciones con distinto nivel de especificidad en términos de las competencias ocupacionales disponibles para el trabajador. Por otro lado, el autor también señaló que un desajuste completo entre el tipo de empleo y el área de estudio implicaba un mayor costo salarial versus un desajuste parcial.

Siguiendo con Robst (2007), este argumentó, además, que la mayor prevalencia de desajuste observada entre egresados de áreas del conocimiento orientadas a la formación de competencias genéricas se explicaba porque este tipo de competencias, comparadas con otras más específicas, se transfieren relativamente a menor costo entre distintos sectores de empleo. De manera inversa, las competencias específicas son más costosas de transferir entre sectores, lo que implica un costo salarial mayor cuando un trabajador formado en 
un área específica se emplea fuera de su área de estudio. Asimismo, el costo salarial del desajuste entre empleo y área de estudio depende de la magnitud del desajuste: los trabajadores empleados en un área totalmente no relacionada con el área de estudio obtienen salarios menores si se los compara con trabajadores empleados en un área que se relaciona de manera parcial con el área de estudio. Al respecto, el autor señaló que en este caso las diferencias en los retornos salariales se explicarían por las distintas intensidades de uso de competencias ocupacionales entre trabajadores enfrentados a variados niveles de desajuste entre empleo y área de estudio.

Es así como, usando datos de una encuesta institucional de egresados de la Universidad Tecnológica Metropolitana de Chile en el período 2014-2017, este trabajo estimó el efecto salarial del desajuste entre el área de estudio y el tipo de empleo, analizando las implicancias de dicho desajuste sobre la percepción de satisfacción con el empleo. En particular, se buscó responder las siguientes preguntas de investigación: ¿Cómo afecta el empleo fuera del área de estudio a los salarios y a la satisfacción laboral? ¿Qué características de las trayectorias educativas y laborales explican la magnitud del desajuste entre área de estudio y tipo de empleo?

\section{Revisión de literatura}

La teoría del capital humano (Becker, 1964; Mincer, 1958) constituye la principal explicación utilizada por las ciencias sociales para entender la relación entre educación superior y empleo (Marginson, 2016). Desde su fundación en la década de 1960, ha estimulado un enorme cuerpo de estudios empíricos en el área de la economía, influenciando fuertemente la colección de datos en la investigación en ciencias sociales (Marginson, 2016). Una de sus principales aplicaciones se encuentra en los estudios de trayectorias laborales, campo en el que domina el trabajo de economistas y sociólogos interesados en cuantificar beneficios pecuniarios y no pecuniarios de la educación superior (Teichler, 1999). Diversos autores identifican la presencia de un sesgo cuantitativo en los temas y métodos usados en los estudios de trayectorias laborales (Psacharopoulos, 1994; Teichler, 1999). Por el lado de las temáticas, dominan las orientadas a 
la identificación de competencias demandadas por el mercado laboral y aquellas encaminadas a la caracterización de trayectorias laborales y su relación con la educación superior. En términos de los métodos empleados, estos típicamente hacen uso de estadísticas y encuestas a estudiantes, egresados, empleadores y actores relevantes del sistema (Teichler, 1999).

La principal aplicación empírica de la teoría del capital humano es la conocida ecuación de Mincer, que relaciona el logaritmo de los salarios con los años de escolaridad, la experiencia laboral y su cuadrado (Hartog, 2000). A partir de ella se ha confirmado el rol del sistema de educación superior como mecanismo promotor de movilidad social (Urzúa, 2012), donde la principal evidencia en esta línea surge de las estimaciones de retornos salariales que muestran a los graduados de instituciones de educación superior obteniendo mayores retornos comparados con egresados de otros niveles educacionales (Montenegro \& Patrinos, 2014). Mientras los egresados de programas de educación superior aparecen como ganadores en la economía moderna (Roksa \& Levey, 2010), los resultados en las líneas de sobreeducación y desajuste educacional muestran que, tras controlar por los años de experiencia laboral, los retornos salariales varían entre trabajadores con el mismo nivel de escolaridad.

Los estudios acerca de la sobreeducación distinguen entre la cantidad de escolaridad obtenida y la requerida en los puestos de trabajo y, a partir de la diferencia entre ambas, se derivan medidas de sobre y subeducación y se estiman los retornos de las diferencias y del calce entre ambas (Hartog, 2000). El método empírico utilizado en los estudios en el área de sobreeducación es atractivo por la simplicidad de la estimación y su interpretación. Robst (2007) sigue este enfoque e introduce una nueva medida de desajuste basada en la diferencia entre el tipo de empleo y el área de estudio. Mientras los estudios relativos a la sobreeducación muestran que los retornos salariales se distancian de la situación óptima cuando la escolaridad del trabajador es diferente a la requerida en el empleo actual, dicho autor muestra que los retornos salariales también son castigados cuando un trabajador se emplea fuera de su área de formación: sus resultados indican que estos últimos acceden a salarios menores 
comparados con trabajadores con igual escolaridad empleados en puestos pertinentes. La magnitud del efecto salarial depende del área de estudio y del grado de desajuste entre esta y el empleo. Los egresados de programas de estudio orientados a la formación de competencias genéricas que se emplean fuera de su área de estudio enfrentan una mayor probabilidad de desajuste entre empleo y área de estudio y, además, están sujetos a un menor costo salarial producto del desajuste, si se los compara con los egresados de programas orientados a la formación de competencias específicas.

Estos resultados sugieren que las decisiones que determinan la asignación de estudiantes entre programas de educación superior poseen una racionalidad económica factible de ser estudiada a través de los premios salariales disponibles en el mercado laboral (Robst, 2007). Aunque la investigación no determina la teoría que mejor explica la existencia de desajustes en el mercado laboral, los estudios son consistentes con teorías que analizan el comportamiento de este desde la perspectiva del capital humano, así como también con aquellas que analizan los procesos de búsqueda y ajuste en el mercado laboral (Hartog, 2000; Robst, 2007). En el modelo de búsqueda, individuos con cierto nivel de escolaridad querrán mejorar su nivel educacional con el objetivo de alcanzar el nivel de escolaridad requerido en sus empleos. A su vez, en el modelo de ajuste, los trabajadores aceptarán los empleos mejor remunerados según su nivel de escolaridad (Hartog, 2000).

El modelo de capital humano ofrece una explicación alternativa para entender la racionalidad económica del desajuste entre empleo y área de estudio. A través del estudio de trayectorias laborales en Estados Unidos, Shaw (1984) formuló una especificación de la ecuación de salarios usando el concepto de inversión en empleabilidad. Bajo este enfoque, los trabajadores acumulan competencias a medida que avanzan en sus trayectorias formativas y laborales. Las competencias ocupacionales son las que un trabajador necesita para desempeñarse en un empleo específico. La intensidad con que estas se usan en un determinado empleo dependerá del grado de ajuste entre empleo y tipo de formación recibida por el trabajador. Quienes trabajan en áreas completamente distintas de las propias 
usarán con menor intensidad sus competencias ocupacionales y enfrentarán un costo salarial mayor, comparado con quienes trabajan en áreas parcial o completamente relacionadas con su área de estudio.

La teoría del capital humano ha sido foco de una fuerte crítica, debido principalmente al supuesto de racionalidad individual asociado con la elección de carrera. En este contexto, los resultados provenientes de la investigación en las áreas de sobreeducación y desajuste educacional advierten que la mayor prevalencia de desajustes en las trayectorias laborales puede significar que el problema de elección de carrera tenga un trasfondo social mucho más relevante que el advertido por la moda de resultados en estudios de retornos salariales (Marginson, 2016; Montenegro \& Patrinos, 2014). Aunque Marginson argumenta que la prevalencia del desajuste entre empleo y área de estudio observada en Estados Unidos podría develar una desconexión entre el sistema de educación superior y el mercado laboral (Marginson, 2016), los resultados de Robst (2007) indican que el efecto salarial es menor en magnitud en áreas más prevalentes, suavizando el costo económico promedio asociado con el problema de elección de carrera.

Con el objetivo de analizar las fuentes de heterogeneidad observadas en los estudios de retornos salariales, Marginson (2016) recopiló una serie de resultados provenientes de estudios de selección laboral y redes de egresados. La recopilación sugiere que los salarios varían en diversos aspectos de las trayectorias educativas de los egresados de programas de educación superior. Algunas de las dimensiones que configuran un escenario heterogéneo en términos de retornos salariales (Bingley, Corak \& Westergard-Nielsen, 2011; Borgen, 2015; Melguizo \& Wolniak, 2012; Rivera, 2011; Roksa \& Levey, 2010; Tholen, Brown, Power \& Allouch, 2013; Wolniak, Seifert, reed \& Pascarella, 2008) son: los ingresos familiares; las habilidades cognitivas y no cognitivas; el tipo de educación secundaria recibida; la institución de educación superior desde donde el postulante se graduó; las redes sociales y familiares del egresado al momento de ingresar a la educación superior; la influencia familiar y de las redes sociales al momento de ingresar al mundo laboral; las redes sociales desarrolladas a través de la carrera; las actividades 
extracurriculares realizadas como estudiante; el capital social de los postulantes; diferencias en estatus y recursos de las instituciones de educación superior; y las áreas del conocimiento.

Marginson (2016) plantea que una de las características del proceso de inserción laboral es que los egresados tienden a tomar los empleos que proveen mejores pagos y expectativas de desarrollo futuro al momento de postulación y selección. A su vez, los empleadores seleccionan a "los mejores" entre los disponibles. De allí que en la selección de egresados en determinados empleos influyen no solo las contingencias del tiempo y lugar, sino también las alternativas disponibles para egresados y empleadores al momento de la selección.

La investigación en las áreas de desajuste educacional y crítica a la teoría del capital humano sugiere algunas hipótesis pertinentes de estudiar a través del seguimiento de trayectorias educativas y laborales. El principal resultado aportado por Robst (2007) corresponde a la identificación del patrón inverso entre riesgo y costo del desajuste entre áreas de estudio con distinto nivel de especificidad en términos de las competencias ocupacionales transmitidas por ellas en sus puestos de trabajo. Este resultado se valida mediante el contraste de dos hipótesis. La primera sugiere que la probabilidad de trabajar fuera del área de estudio es mayor entre egresados de programas orientados a la formación de competencias ocupacionales genéricas o transversales, comparada con egresados de programas con mayor especificidad de competencias. La racionalidad de esta afirmación se basa en la noción de que las competencias genéricas se transfieren a bajo costo entre empleos ubicados en distintas áreas del conocimiento, comparadas con las competencias ocupacionales con mayor nivel de especificidad que, en esencia, son más difíciles de transferir entre distintos tipos de empleo. La segunda hipótesis sugiere que los salarios de egresados que trabajan fuera del área de estudio son menores a los obtenidos por egresados con el mismo nivel de escolaridad y experiencia laboral que se emplean en puestos de trabajo pertinentes. Sin embargo, se podría aventurar una tercera hipótesis que sugiere que el desajuste entre empleo y área de estudio debiera ejercer un impacto negativo sobre la percepción de satisfacción con el 
empleo. Esta última hipótesis no ha sido contrastada en la literatura y este trabajo muestra evidencia que afirma la existencia de una relación inversa entre satisfacción y desajuste.

\section{Los datos}

La base de datos se construyó a partir de la consolidación de cuatro versiones de una encuesta anual de empleabilidad de egresados de una universidad estatal metropolitana (2014-2017). En cada año de aplicación se encuestaron dos cohortes de titulación, a saber, a quienes recibieron su título profesional hacía un año (inserción laboral) y a quienes lo recibieron hacía tres años (progresión laboral). Con ello, la base de datos contempló cuatro años de información para cada cohorte de titulación, es decir, los titulados en período de inserción egresan entre 2013 y 2016, en tanto que los titulados en el período de progresión lo hacen entre 2011 y 2014. El crecimiento del tamaño muestral generado por la consolidación de cuatro versiones de la encuesta buscó mejorar la precisión de las pruebas estadísticas aplicadas a los datos. La Tabla 1 contiene la distribución de la muestra según año de egreso, etapa de la trayectoria laboral analizada y área del conocimiento de la carrera de origen, incorporando en cada caso el porcentaje de mujeres presentes en la muestra.

La mayor presencia de egresados del área del conocimiento de Tecnología hace que esta categoría haya sido utilizada como línea de base en las comparaciones estadísticas. Por otro lado, aunque la muestra completa se encuentra balanceada entre hombres y mujeres, esto no ocurrió en ciertas áreas del conocimiento. El porcentaje de mujeres que escogen el área Tecnología (35\%) contrasta con el observado en el área Ciencias Sociales y Humanidades (77\%). La expectativa de barreras de entrada a cierto tipo de empleos puede afectar la elección de carrera, particularmente en el caso de las mujeres (Polachek, 1978). 


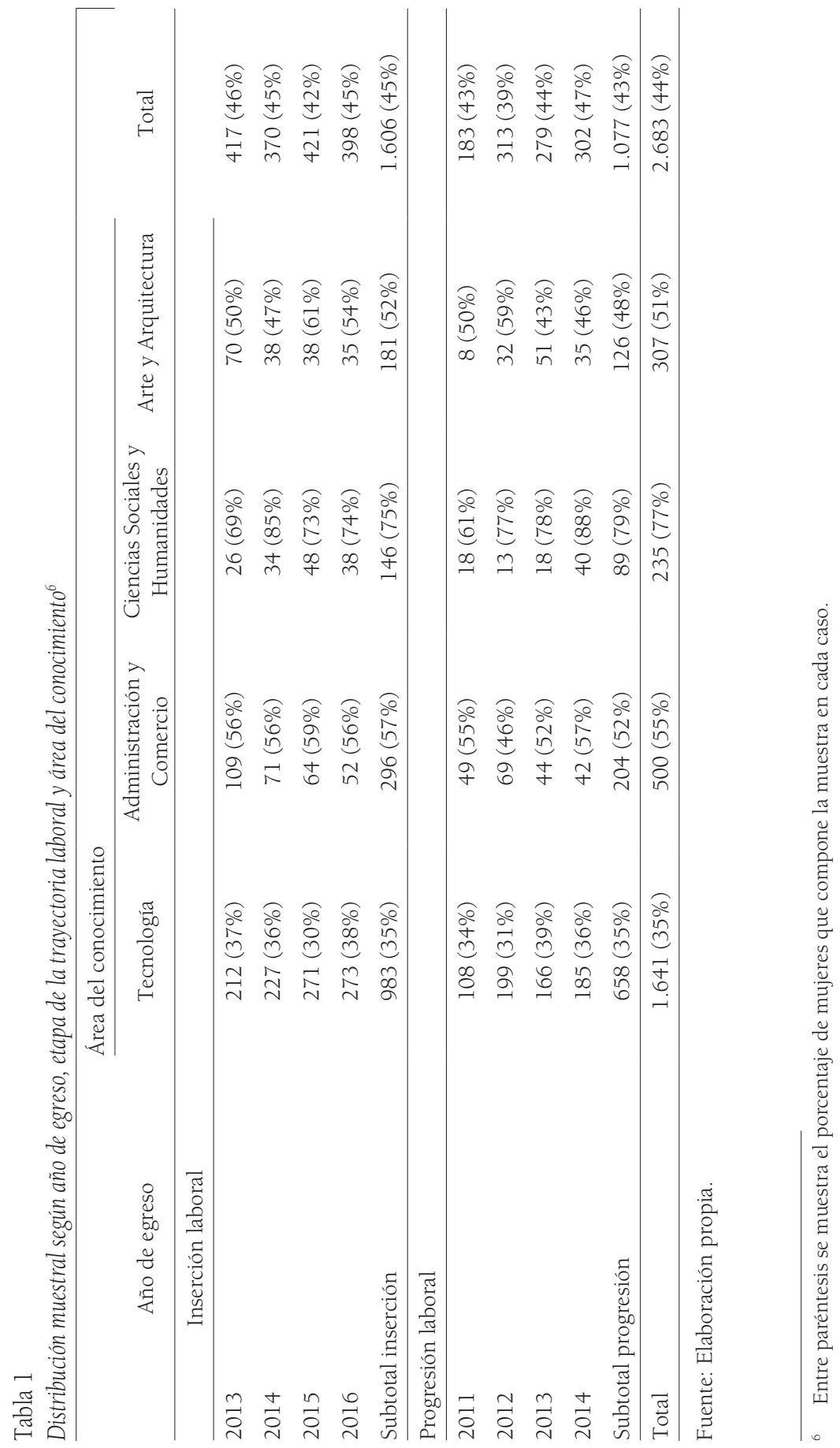


El instrumento de empleabilidad utilizado en la encuesta indaga diversos aspectos de las trayectorias laborales, tales como: experiencia laboral (cantidad de empleos a la fecha); características del primer empleo a un año de haber egresado (pertinencia, redes y tiempo de búsqueda); características del empleo actual a uno y tres años de haber egresado (situación laboral, redes de búsqueda, pertinencia, sector económico, jornada laboral, tipo de contrato, cargo u ocupación, salarios, ingresos y niveles de satisfacción con el empleo); razones del desempleo; y nivel de relevancia de ciertas competencias profesionales en el desempeño laboral.

Las preguntas de la encuesta que permiten estimar el costo salarial del desajuste entre el empleo actual y la carrera de origen son las siguientes:

- Su trabajo actual, ¿itiene relación con la carrera que usted estudió?

- Para trabajadores dependientes: ¿Cuál es aproximadamente su remuneración líquida mensual?

- Para trabajadores independientes: En un mes promedio, ¿cuál es el ingreso líquido aproximado de su trabajo?

A su vez, las preguntas de la encuesta que permiten analizar las brechas de satisfacción con el empleo actual originadas por el desajuste entre empleo y carrera de origen son las siguientes:

- Evalúe qué tan satisfecho está con su actual trabajo en una escala de 1 a 7 , donde 1 es Nada satisfecho y 7 es Muy satisfecho.

- [Pregunta abierta] ¿Por qué califica con esta nota?

\section{Metodología}

Los determinantes de la probabilidad de desajuste entre área de estudio y tipo de empleo se estimaron mediante el método Probit, que es una regresión probabilística que usa una variable dependiente binaria que indica si el egresado trabaja en un empleo no relacionado con la carrera de origen:

$$
P(d)_{i j k}=X_{i j k} \beta+Z_{j} \rho+\varepsilon_{i},(1)
$$


donde $X$ incluye un vector de variables socioeconómicas del egresado $i$, un vector de características de la trayectoria educacional del egresado $i$ en el área del conocimiento $j$, y un vector de características del empleo actual que considera el sector económico $k$ al que pertenece el puesto de trabajo, el mecanismo de búsqueda de empleo usado por el egresado, y otras características de la trayectoria laboral del egresado. A su vez, Z es una variable categórica binaria que identifica el área del conocimiento de la carrera de origen del egresado. La variable $Z$ es igual a cero cuando el área de conocimiento es Tecnología y es igual a uno en los otros casos. La variable didentifica si la persona trabaja en un empleo no relacionado con su área de estudio. La identificación del desajuste entre área de estudio y tipo de empleo se realiza a partir del reporte del encuestado, dada la construcción binaria de desajuste planteada por la encuesta entre las categorías completa o nula.

El costo salarial de estar empleado en un área no relacionada con la carrera estudiada se estimó por mínimos cuadrados ordinarios, una ecuación de salarios estándar donde el logaritmo del salario queda explicado por:

$$
\operatorname{Ln}\left(w_{i j k}\right)=X_{i j k} \beta+Z_{j} \alpha+d_{i j} \mu+\varepsilon_{i},(2)
$$

donde $X, Z$ y d se definen del mismo modo que en (1).

Para conocer los determinantes del nivel de satisfacción con el empleo actual se usó el método Logit ordenado que es una regresión probabilística que explica los niveles de satisfacción alto, medio y bajo:

$$
P(\text { satisfacción alta })_{i}=X_{i j k} \beta+Z_{j} \rho+d_{i j} \gamma+\mu_{i},(3)
$$

donde $Z$ y d se definen del mismo modo que en (1) y ahora en $X$ adicionalmente se incorpora el salario como variable explicativa.

La hipótesis que sugiere que la probabilidad de trabajar fuera del área de estudio es mayor entre egresados de programas distintos al área de conocimiento Tecnología, se contrasta estimando $\rho$ en la ecuación (1). Se espera que $\rho$ tenga signo positivo para las áreas del conocimiento distintas al área Tecnología, que se asumen relacionadas con programas orientados a la formación de competencias genéricas. 
La hipótesis que sugiere que los salarios de egresados que trabajan fuera del área de estudio son menores a los obtenidos por egresados con el mismo nivel de escolaridad y experiencia laboral que se emplean en puestos de trabajo pertinentes, se contrasta estimando $\mu$ en (2). Se espera que $\mu$ tenga signo negativo, evidenciando el signo esperado del diferencial salarial entre trabajadores empleados fuera de su área de estudio comparado con trabajadores empleados en puestos pertinentes.

La hipótesis que sugiere que el desajuste entre empleo y área de estudio debiera ejercer un impacto negativo sobre la percepción de satisfacción con el empleo, se contrasta por dos vías, una estadística y otra semántica. La vía estadística se resuelve a través de la estimación de $\gamma$ en la ecuación (3). Se espera que $\gamma$ posea signo negativo, evidenciando que el desajuste entre empleo y área de estudio afecte negativamente la probabilidad de estar altamente satisfecho con el empleo actual. Esta hipótesis también se evalúa mediante la técnica de análisis semántico, aplicado sobre las respuestas de los egresados frente a la pregunta abierta que indaga en las razones asociadas a su nivel de satisfacción con el empleo actual. El análisis semántico operó por la vía del análisis de palabras clave y el análisis de concordancias. El análisis de palabras clave permite descubrir cuáles son aquellas palabras que, desde un punto de vista estadístico, son significativamente más usadas por un grupo respecto de otro con el cual se está comparando. En otras palabras, permite dilucidar aquello que es propio en el habla de un grupo en relación con otro. A su vez, las colocaciones son palabras que, desde un punto de vista estadístico, tienden a ocurrir juntas en un texto. El estadístico de colocaciones tiende a privilegiar más las palabras léxicas que las gramaticales, en la medida en que se privilegia la exclusividad de la relación entre las palabras (esto es, que la palabra de la colocación no ocurra con otras palabras) por sobre la frecuencia de la colocación (Mautner, 2015).

Considerando la existencia de importantes diferencias de género en términos de elección de carrera y profesión, todas las ecuaciones se estiman de forma separada para hombres y mujeres (Polachek, 1978, 1981), así como también para egresados que se encuentran a uno y tres años de haber egresado de la universidad. 
A su vez, el análisis semántico se realiza de manera separada para hombres y mujeres en las etapas de inserción y progresión laboral, por una parte, e igualmente se analizan por separado las respuestas correspondientes a egresados que reportan desajuste completo versus aquellos que reportan desajuste nulo entre empleo y área de estudio.

\section{Resultados}

\subsection{Caracterización de trayectorias educativas}

La Tabla 2 describe las trayectorias educativas de los egresados que componen la muestra, por área del conocimiento y duración de las carreras. Las trayectorias se describen según variables que caracterizan la progresión académica de los egresados:

- semestres de sobreduración, calculado como la diferencia en semestres entre la duración efectiva y la duración teórica de la carrera;

- nota final de egreso de la carrera;

- tasa de aprobación de asignaturas durante el primer y segundo año de carrera;

- puntaje de ingreso PSU promedio Lenguaje y Matemática; y

- porcentaje de egresados cuyos padres poseen educación superior completa. 


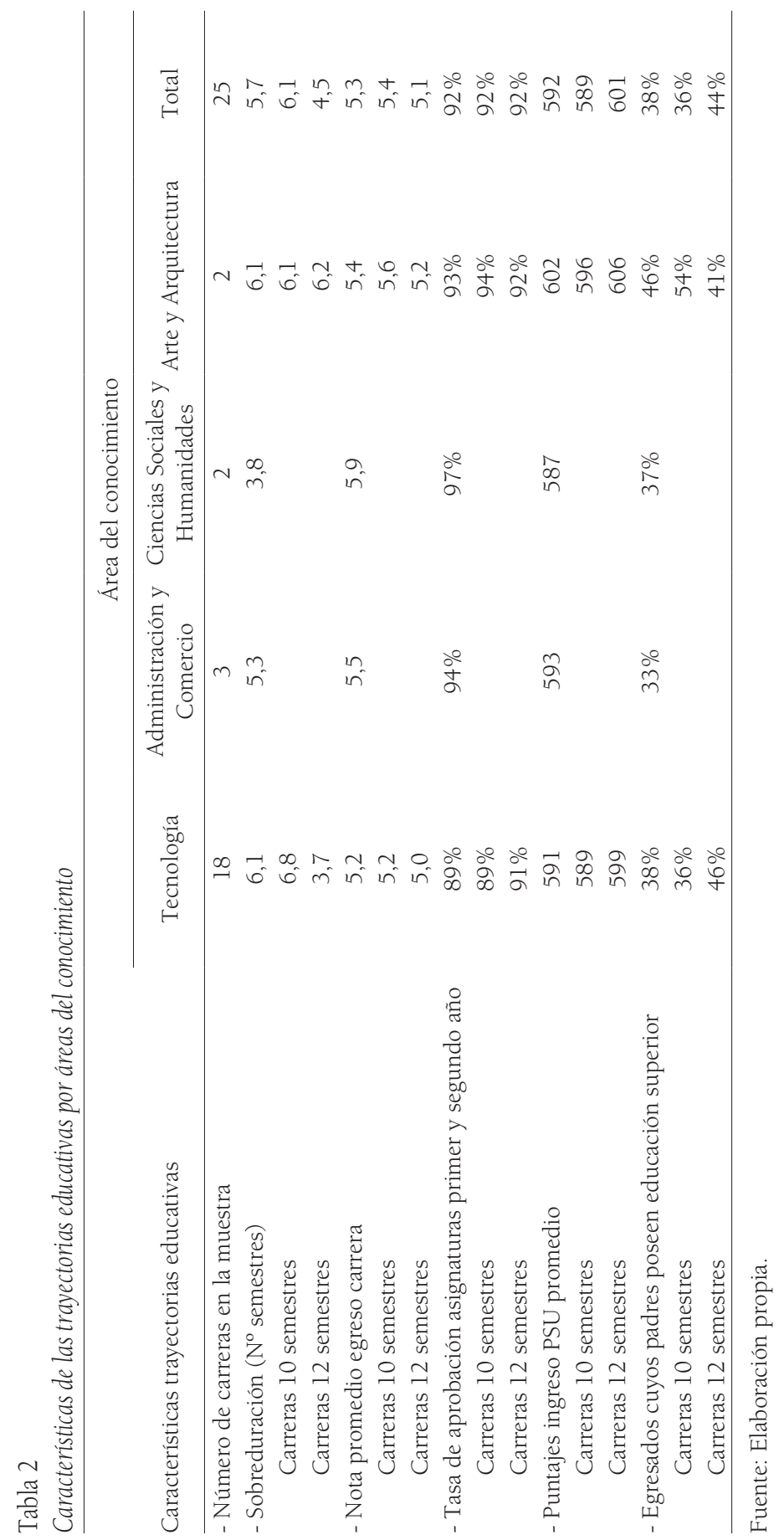


La Figura 1 muestra tipos de correlaciones entre los valores promedio de las variables que caracterizan las trayectorias educativas ${ }^{7}$. En primer lugar, se observa una alta correlación entre la tasa de aprobación de asignaturas de primer año, el promedio de notas de egreso, y los semestres de sobreduración, todas variables que caracterizan la progresión académica de los egresados. En aquellas áreas del conocimiento que presentan altas (bajas) tasas de aprobación de asignaturas en el primer año, se observan mayores (menores) promedios de egreso y menores (mayores) niveles de sobreduración de carreras. Las carreras de 12 semestres del área Tecnología representan la excepción a la regla con bajos promedios finales de egreso y sobreduración, respectivamente. En segundo lugar, se observa una correlación entre el porcentaje de egresados cuyos padres o madres poseen educación superior completa y los puntajes de ingreso promedio obtenidos en la PSU. Por último, el perfil socioeconómico de puntajes de ingreso a la universidad no se correlaciona con la eficacia en la progresión académica de los egresados, medida a través del indicador de sobreduración.

Cada punto en el plano representa la intersección de los promedios de cada variable por área del conocimiento y duración de carreras. CS10: carreras de 10 semestres de duración del área de las Ciencias Sociales; AC10: carreras de 10 semestres de duración del área de Administración y Comercio; AA10: carreras de 10 semestres de duración del área de Arte y Arquitectura; AAl2: carreras de 12 semestres de duración del área de Arte y Arquitectura; T10: carreras de 10 semestres de duración del área de Tecnología; T12: carreras de 12 semestres de duración del área de Tecnología. 

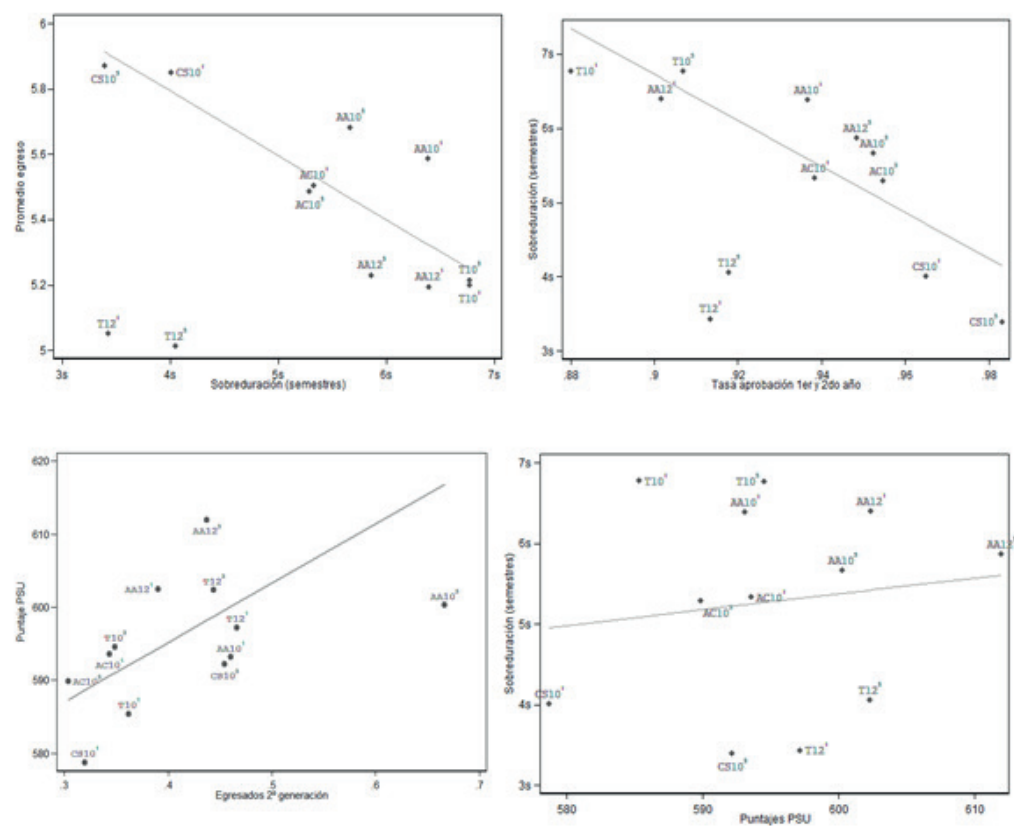

Figura 1. Descripción de trayectorias educativas por área del conocimiento.

Fuente: Elaboración propia.

Mientras las carreras de 10 semestres del área de Tecnología y las de 12 semestres del área Arte y Arquitectura enfrentan tasas de sobreduración mayores comparadas con carreras de 10 semestres de duración de las áreas de las Ciencias Sociales y Humanidades y de Administración y Comercio, respectivamente, además sus promedios de egreso y avance curricular inicial de la carrera son menores.

\subsection{Caracterización del desajuste}

La Tabla 3 muestra la incidencia del desajuste entre empleo y área de estudio según las etapas de la trayectoria laboral de los egresados, su sexo, área del conocimiento de la carrera de origen y sector económico del lugar de trabajo. Entre los egresados que se encuentran a un año de haber egresado, un $8 \%$ reporta poseer un empleo no relacionado con su área de estudio (10\% entre las mujeres y $7 \%$ entre los hombres). A su vez, entre quienes se encuentran a tres años de haber egresado, un $9 \%$ reporta poseer un empleo no 
relacionado con su área de estudio (sin diferencias según sexo). En relación con las áreas del conocimiento, el desajuste es más frecuente en las mujeres egresadas de las áreas de Tecnología y Ciencias Sociales y Humanidades y, en el caso de los hombres, entre los egresados del área de Administración y Comercio. A su vez, el desajuste es nulo en el caso de los hombres egresados de carreras de Ciencias Sociales y Humanidades. En términos del sector económico del empleo actual, el desajuste es más frecuente entre las mujeres a un año de haber egresado que se encuentran empleadas en los sectores de Servicios, Comercio, y Tecnología y Telecomunicaciones; así como también entre las mujeres que se encuentran a tres años de haber egresado y se emplean en el sector Financiero. En el caso de los hombres que se encuentran a un año de haber egresado, el desajuste es más frecuente entre quienes se emplean en el sector Transportes; y en el caso de los hombres que se encuentran a tres años de haber egresado, el desajuste es más frecuente entre quienes se emplean en los sectores de Servicios y Comercio. El desajuste es nulo en el caso de los hombres empleados en el sector Construcción. 


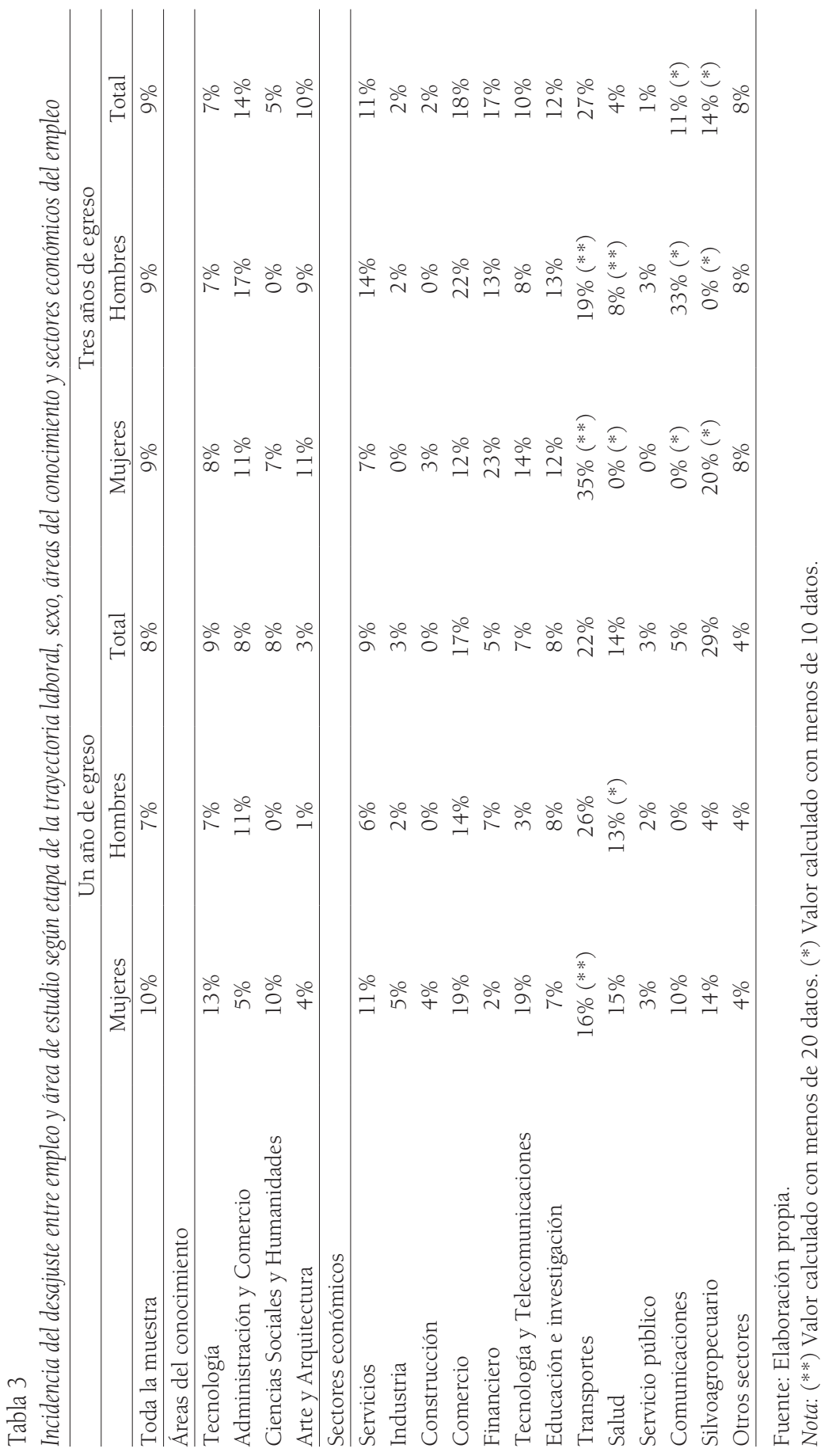




\subsection{Probabilidad de desajuste según área del conocimiento}

La Tabla 4 contiene los resultados de los modelos de probabilidad de desajuste entre empleo y áreas de estudio. Entre las mujeres a un año de haber egresado, el desajuste es menos probable entre quienes egresan de las áreas de Administración y Comercio, y Arte y Arquitectura, en comparación con sus pares de las áreas de Tecnología y Ciencias Sociales, respectivamente. Asimismo, las mujeres a un año de haber egresado empleadas en los sectores Comercio, y Tecnología y Telecomunicaciones, respectivamente, enfrentan una mayor probabilidad de desajuste entre empleo y área de estudio. Por otro lado, entre los hombres a un año de haber egresado, no hay diferencias según áreas del conocimiento, excepto en el área Ciencias Sociales y Humanidades donde todos los hombres se emplean en puestos pertinentes. En términos del sector económico del empleo actual, los hombres empleados en el sector Transporte enfrentan una mayor probabilidad de desajuste, mientras que todos los hombres empleados en el sector Construcción lo hacen en puestos pertinentes.

Entre las mujeres a tres años del egreso, la probabilidad de desajuste no varía según área del conocimiento, con la excepción al alza en el área Arte y Arquitectura, aunque sí aumenta según sector económico del empleo actual, particularmente en los sectores Financiero e Industria. En el caso de los hombres a tres años del egreso, se observa una mayor probabilidad de desajuste en el área de conocimiento de Administración y Comercio, mientras todos los egresados del área Ciencias Sociales y Humanidades se emplean en puestos pertinentes.

Los modelos incluyen varios tipos de variables de control. En términos de las características de las trayectorias universitarias, las mujeres y hombres cuyo tiempo de titulación excede el tiempo de titulación promedio por carrera enfrentan una mayor probabilidad de desajuste a un año de haber egresado. En comparación con la categoría omitida correspondiente a carreras de 10 semestres de duración, las mujeres y hombres que egresan de carreras de 12 semestres de duración enfrentan una menor probabilidad de desajuste. En comparación con la variable omitida correspondiente al método de búsqueda de empleo mediante postulación abierta o concurso, las mujeres empleadas a 
través de una práctica profesional poseen una menor probabilidad de desajuste a un año de haber egresado, mientras que los hombres que consiguieron empleo a través de un conocido en la empresa o un familiar poseen una mayor probabilidad de desajuste a tres años de haber egresado. En comparación con el empleo dependiente, las mujeres que trabajan de manera independiente poseen una mayor probabilidad de desajuste a un año de haber egresado. En cuanto al empleo jornada completa, los hombres que trabajan media jornada enfrentan una mayor probabilidad de desajuste. Por último, la antigüedad en el empleo disminuye la probabilidad de desajuste en los hombres a un año de haber egresado.

Tabla 4

Resultados de modelos de probabilidad de desajuste entre empleo y áreas de estudio

\begin{tabular}{|c|c|c|c|c|}
\hline \multirow{2}{*}{$\begin{array}{l}\text { Variable dependiente: Probabilidad de } \\
\text { desajuste } \\
\text { Variables explicativas }\end{array}$} & \multicolumn{2}{|c|}{ Un año de egreso } & \multicolumn{2}{|c|}{ Tres años de egreso } \\
\hline & Mujeres & Hombres & Mujeres & Hombres \\
\hline (i) Administración y Comercio & $-0,050 * *$ & & & $0,068^{*}$ \\
\hline (i) Ciencias Sociales y Humanidades & & $\mathrm{Pp}(-)$ & & $\mathrm{Pp}(-)$ \\
\hline (i) Arte y Arquitectura & $-0,060 * * *$ & & $0,155^{*}$ & \\
\hline (ii) Sobreduración & $0,007 *$ & $0,004 *$ & & \\
\hline (ii) Carreras de 12 semestres & $-0,054 * *$ & & $-0,075 * *$ & $-0,058^{* *}$ \\
\hline (iii) Sector Comercio & $0,159 * *$ & & & \\
\hline $\begin{array}{l}\text { (iii) Sector Tecnología y } \\
\text { Telecomunicaciones } \\
\text { (iii) Sector Financiero }\end{array}$ & $0,139 *$ & $-0,045^{* *}$ & $0,232 *$ & \\
\hline (iii) Sector Industria & & $-0,045^{* *}$ & & $-0,071^{* * *}$ \\
\hline (iii) Sector Transportes & & $0,197^{*}$ & $0,336 * *$ & \\
\hline (iii) Sector Construcción & & $\operatorname{Pp}(-)$ & & $\mathrm{Pp}(-)$ \\
\hline (iii) Sector Público & & $-0,043^{*}$ & & \\
\hline (iv) Práctica profesional & $-0,073 * * *$ & & & \\
\hline (iv) A través de un conocido en la empresa & & & & $0,071^{*}$ \\
\hline (iv) A través de un familiar & & & & $0,099 *$ \\
\hline (v) Empleo independiente & 0,081 & & & \\
\hline (v) Media jornada & & 0,126 & & \\
\hline (v) Antigüedad en el empleo & & $-0,001^{* *}$ & & \\
\hline $\mathrm{N}$ observaciones & 533 & 647 & 372 & 541 \\
\hline Adj. $R^{2}$ & $14 \%$ & $13 \%$ & $14 \%$ & $10 \%$ \\
\hline Desajuste observado vs predicción & $9 \%$ vs $6 \%$ & $7 \%$ vs $5 \%$ & $9 \%$ vs $7 \%$ & $9 \%$ vs $7 \%$ \\
\hline
\end{tabular}

Fuente: Elaboración propia.

Nota: ${ }^{* *}$ significativa al 1\%; ** significativa al 5\%, * significativa al 10\%. (i) Áreas del conocimiento, (ii) Características trayectoria universitaria, (iii) Sector económico empleo actual, (iv) Redes de búsqueda de empleo, (v) Características trayectoria laboral. $\mathrm{Pp}(-$ ) significa que la variable explicativa predice perfectamente el desajuste. 


\subsection{Efectos del desajuste sobre los retornos salariales}

\subsubsection{Retornos a la escolaridad}

La Tabla 5 muestra los resultados de los modelos que identifican el efecto salarial del desajuste entre empleo y área de estudio. Las mujeres y hombres que se emplean en puestos no relacionados con su área de estudio obtienen salarios menores que los trabajadores que reportan hacerlo en un empleo que sí se vincula con su área de estudio. El efecto marginal es mayor entre hombres y mujeres que se encuentran a tres años de haber egresado, comparado contra quienes lo hicieron hace un año. Esto significa que el empleo en un área no relacionada con la carrera de origen disminuye la tasa de retorno a la escolaridad en hombres y mujeres.

Los modelos incluyen varios tipos de variables de control. En comparación con los salarios de egresados de la categoría omitida correspondiente al área del conocimiento de Tecnología, los salarios de mujeres y hombres egresados de las áreas del conocimiento de las Ciencias Sociales y Humanidades y Arte y Arquitectura son menores. En comparación con los salarios de egresados de la categoría omitida correspondiente a carreras de 10 semestres de duración, los salarios de mujeres y hombres que egresan de carreras de 12 semestres de duración son mayores. En comparación con los salarios de egresados que se emplean en el sector Servicios, los salarios de mujeres y hombres que se emplean en los sectores Tecnología y Telecomunicaciones, Financiero, Industria, Construcción y Sector Público, son respectivamente mayores. En comparación con los salarios de los trabajadores con empleo dependiente, los hombres que lo hacen de manera independiente ganan salarios menores y los microempresarios, mayores. A su vez, la antigüedad en el empleo actual y el número de empleos previos incrementan los salarios de hombres y mujeres. Por último, en el caso de las mujeres a un año de haber egresado, el ciclo económico afecta positivamente los salarios en los años 2015, 2016 y 2017. 
Tabla 5

Efectos salariales del desajuste entre empleo y área de estudio

\begin{tabular}{|c|c|c|c|c|}
\hline \multirow{2}{*}{$\begin{array}{l}\text { Variable dependiente: } \operatorname{Ln}(w) \\
\text { Variables explicativas }\end{array}$} & \multicolumn{2}{|c|}{1 año de egreso } & \multicolumn{2}{|c|}{3 años de egreso } \\
\hline & Mujeres & Hombres & Mujeres & Hombres \\
\hline Desajuste & $-0,180 * *$ & $-0,140 *$ & $-0,261 * * *$ & $-0,296^{* * *}$ \\
\hline (i) Administración y Comercio & $0,076^{*}$ & & & \\
\hline (i) Ciencias Sociales y Humanidades & $-0,169 * * *$ & $-0,232 * *$ & $-0,234 * * *$ & $-0,468 * * *$ \\
\hline (i) Arte y Arquitectura & $-0,194 * * *$ & $-0,289 * * *$ & $-0,115^{*}$ & $-0,242 * * *$ \\
\hline (ii) Carreras de 12 semestres & $0,240 * * *$ & $0,126 * * *$ & $0,245^{* * *}$ & $0,162 * * *$ \\
\hline $\begin{array}{l}\text { (iii) Sector Tecnología y } \\
\text { Telecomunicaciones }\end{array}$ & $0,136^{*}$ & & $0,343^{* * *}$ & $0,151^{* *}$ \\
\hline (iii) Sector Financiero & $0,193 * * *$ & $0,174 * * *$ & $0,197^{*}$ & $0,139 * *$ \\
\hline (iii) Sector Industria & $0,105^{*}$ & $0,068^{*}$ & & \\
\hline (iii) Sector Construcción & $0,223 * * *$ & & & \\
\hline (iii) Sector Público & $0,243 * * *$ & $0,138^{* *}$ & $0,162 * *$ & $0,118^{*}$ \\
\hline (iv) Práctica profesional & & & $0,127^{*}$ & \\
\hline (iv) Empleo independiente & & & & $-0,249 *$ \\
\hline (iv) Microempresario & & & & $0,273^{*}$ \\
\hline (v) Media jornada & $-0,201^{* *}$ & $-0,489 * * *$ & $-0,498 * * *$ & $-0,387^{* *}$ \\
\hline (v) Antigüedad en el empleo & $0,004 * * *$ & $0,004 *$ & & $0,004 * * *$ \\
\hline (v) Número de empleos previos & $0,042 * *$ & $0,041^{*}$ & & $0,046 * * *$ \\
\hline Año 2015 & $0,126^{* *}$ & & & \\
\hline Año 2016 & $0.146^{* * *}$ & & & \\
\hline Año 2017 & $0,06^{*}$ & & & \\
\hline $\mathrm{N}$ observaciones & 450 & 491 & 276 & 362 \\
\hline Adj. $R^{2}$ & $31 \%$ & $23 \%$ & $34 \%$ & $31 \%$ \\
\hline
\end{tabular}

Fuente: Elaboración propia.

Nota: *** significativa al 1\%; ** significativa al 5\%, * significativa al 10\%. (i) Áreas del conocimiento, (ii) Características trayectoria universitaria, (iii) Sector económico empleo actual, (iv) Redes de búsqueda de empleo, (v) Características trayectoria laboral.

\subsubsection{Análisis de distribuciones}

La Figura 2 compara las distribuciones de ingreso de los egresados de carreras de 10 semestres, tanto si se emplean en puestos pertinentes o no. El análisis de distribuciones de ingreso muestra que el desajuste entre empleo y área de estudio se concentra en tramos de ingreso bajo tanto en hombres como mujeres a un año de haber egresado, $y$ entre las mujeres a tres años de haber egresado. Asimismo, el desajuste se concentra en tramos de ingresos medios en el caso de los hombres a tres años de haber egresado. Si la percepción de desajuste dependiera inversamente del salario, se produciría endogeneidad en el modelo de salarios y el costo salarial estaría sobrestimado. Pero el tamaño del sesgo depende de la formación de 
expectativas salariales en las respectivas profesiones. En esta línea, la evidencia para Chile señala que la sobreestimación de los salarios futuros se concentra en estudiantes de estratos socioeconómicos bajos y con bajo rendimiento académico (Hastings, Neilson \& Zimmerman, 2015), quienes a su vez poseen bajas probabilidades de titulación. De esta manera, un modelo de salarios de egresados universitarios que incorpora variables que afectan la formación de expectativas salariales, tales como las áreas del conocimiento y el sector económico del empleo actual, atenúa los potenciales sesgos atribuibles a la formación de expectativas salariales.
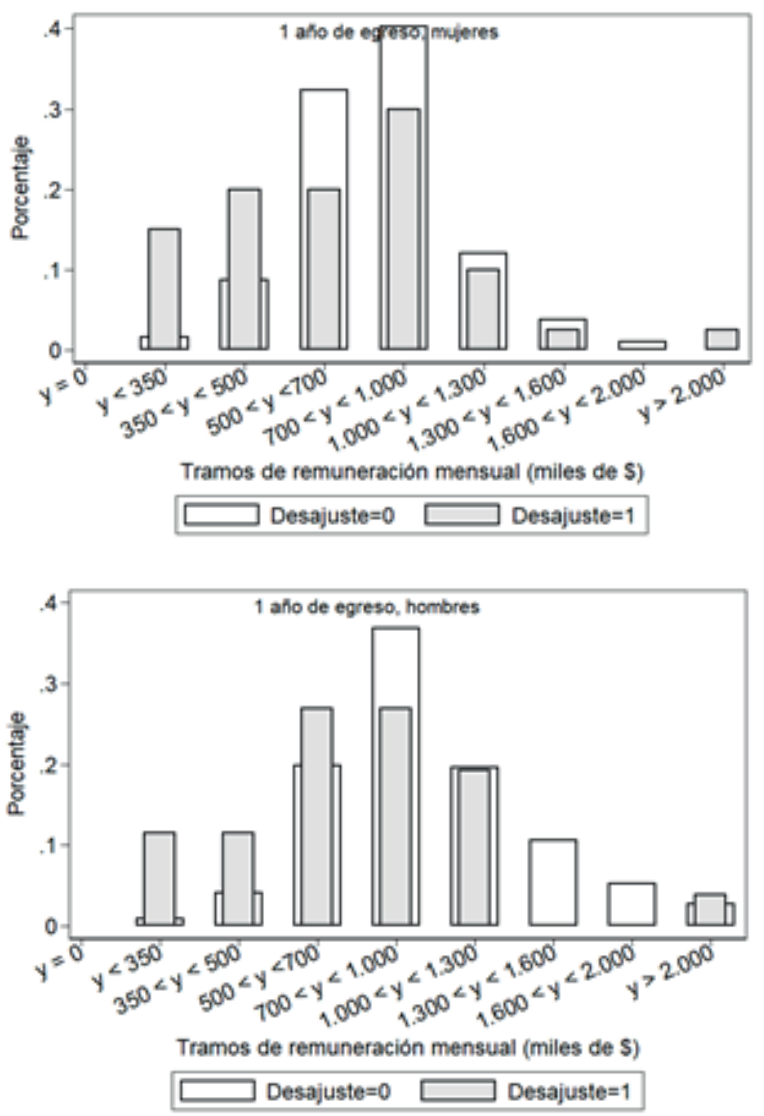

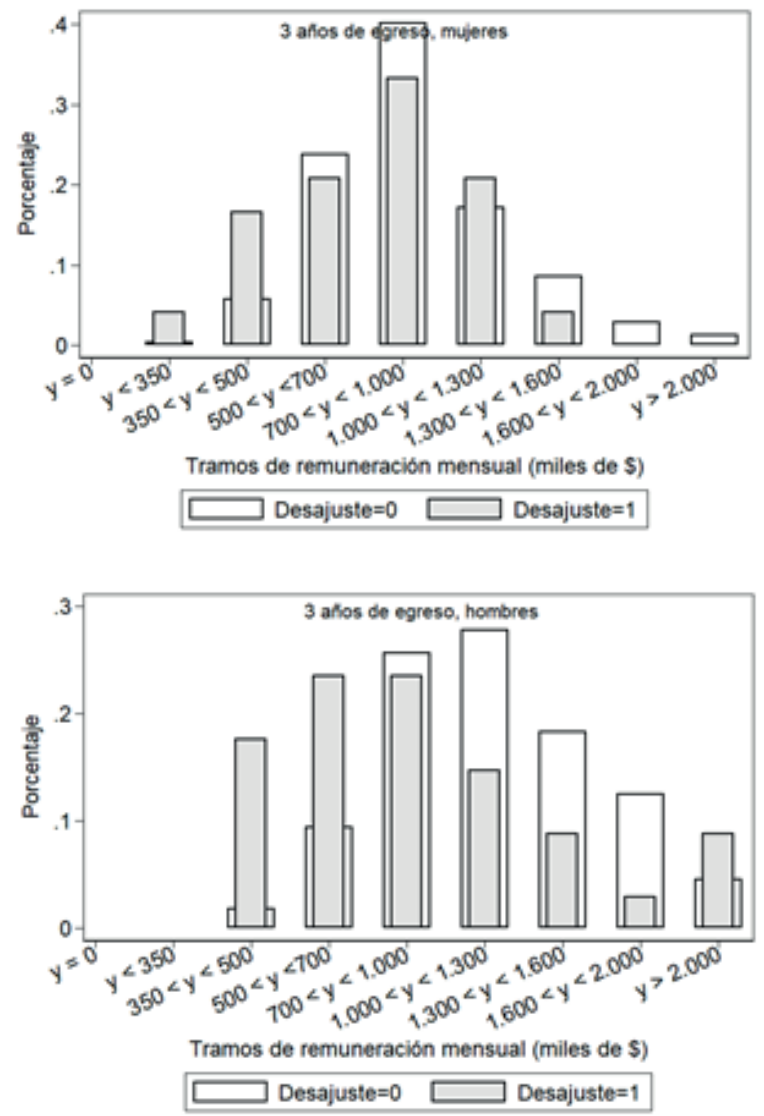

Figura 2. Distribución del ingreso de graduados ajustados y desajustados (según tramos de ingreso mensual en miles de pesos).

Fuente: Elaboración propia.

4.5. Efectos del desajuste sobre la satisfacción con el empleo

4.5.1. Análisis estadístico

La Tabla 6 muestra los resultados de los modelos estadísticos que identifican el efecto del desajuste entre empleo y área de estudio sobre la satisfacción con el empleo actual. La variable dependiente corresponde a la probabilidad de estar altamente satisfecho con el empleo actual, donde la categoría de satisfacción alta se compara con quienes declaran un nivel de satisfacción baja con el empleo actual. 
Un aumento de la probabilidad de estar altamente satisfecho con el empleo actual se relaciona principalmente con el salario, tanto en mujeres como en hombres que se encuentran a un año de haber egresado. No obstante, para los hombres que se están a tres años de haber egresado, el salario no tiene una relación estadísticamente significativa sobre la probabilidad de estar altamente satisfecho con el empleo, y sí la tiene el poseer un empleo no relacionado con el área de estudio, situación que afecta de manera negativa la satisfacción con el empleo actual. En comparación con la categoría omitida correspondiente al área del conocimiento de Tecnología, mujeres y hombres que egresan de carreras relacionadas con las áreas del conocimiento en Administración y Comercio, Ciencias Sociales y Humanidades y Arte y Arquitectura poseen una mayor probabilidad de estar altamente satisfechos con el empleo actual. Por último, en comparación con los hombres que consiguieron empleos mediante postulaciones abiertas o concursos, aquellos que consiguieron empleos a través de un conocido en la empresa o a través de un familiar, poseen una menor probabilidad de estar altamente satisfechos con el empleo actual.

Tabla 6

Resultados de modelos de probabilidad de satisfacción alta con el empleo actual

\begin{tabular}{lcccc}
\hline \multirow{2}{*}{$\begin{array}{l}\text { Variable dependiente: Probabilidad } \\
\text { satisfacción alta }\end{array}$} & \multicolumn{2}{c}{ Un año de egreso } & \multicolumn{2}{c}{ Tres años de egreso } \\
\cline { 2 - 5 } Variables explicativas & Mujeres & Hombres & Mujeres & Hombres \\
\hline $\begin{array}{l}\text { Desajuste } \\
\text { Salario }\end{array}$ & $0,244^{* * *}$ & $0,133^{* *}$ & $0,173^{*}$ & $-0,191^{*}$ \\
(i) Administración y Comercio & $0,168^{* * *}$ & $0,116^{*}$ & & \\
(i) Ciencias Sociales y Humanidades & $0,183^{* * *}$ & & $0,165^{*}$ & \\
(i) Arte y Arquitectura & & & $0,152^{*}$ & \\
(iv) A través de un conocido en la empresa & & $-0,149^{* *}$ & & $-0,241^{* *}$ \\
(iv) A través de un familiar & & & & 403 \\
N observaciones & 398 & 493 & 263 & $403 \%$ \\
Adj. R & $7 \%$ & $6 \%$ & $6 \%$ & $3 \%$ \\
P(obs) vs Predicción & $73 \%$ vs & $73 \%$ vs & $68 \%$ vs & $78 \%$ vs \\
\hline
\end{tabular}

Fuente: Elaboración propia.

Nota: *** significativa al 1\%; ** significativa al 5\%, * significativa al 10\%. (i) Áreas del conocimiento, (ii) Características trayectoria universitaria, (iii) Sector económico empleo actual, (iv) Redes de búsqueda de empleo, (v) Características trayectoria laboral. 


\subsubsection{Análisis semántico}

El análisis semántico de las respuestas que los egresados emiten frente a la pregunta abierta que indaga en las razones asociadas al nivel de satisfacción con el empleo actual, se realizó sobre distintos segmentos de análisis que se indican a continuación. En primer lugar, se identificaron las palabras clave que son significativamente más usadas por los egresados en la etapa de inserción laboral respecto de los egresados en la etapa de progresión laboral y viceversa. En el primer caso, emerge con mayor frecuencia la palabra "quiero". El análisis de concordancia de la palabra "quiero" refiere al deseo de un cambio en el trabajo actual, ya sea en otra área, o donde existan mayores desafíos, remuneraciones u opciones de crecer. A su vez, los egresados en la etapa de progresión laboral usan significativamente más la palabra "porque", indicando una mayor inclinación a fundamentar el nivel de satisfacción con el empleo actual. El análisis de concordancia de la palabra "porque" refiere principalmente a características del empleo que pueden tener una connotación asociada a satisfacción positiva o negativa. Considerando esta evidencia, se identificaron las palabras clave que son significativamente más usadas por los egresados que reportan satisfacción alta con el empleo actual respecto de los egresados que no reportan satisfacción alta y viceversa. En el primer caso emergen conceptos como "ambiente laboral", "horario", "beneficios", y en el segundo caso palabras como "remuneración" y "ganar". También se identificaron diferencias semánticas en las percepciones de satisfacción con el empleo actual entre quienes reportan tener un empleo relacionado con el área de estudio y quienes reportan no tener un empleo relacionado y viceversa. En el primer caso emergieron las palabras "no", "área", "estudié"; mientras que en el segundo caso aparecen las palabras "cosas" y "hago". Considerando la evidencia levantada mediante el análisis estadístico en la línea de satisfacción laboral, se identificaron las palabras clave que son significativamente más usadas por hombres respecto de las mujeres en la etapa de progresión laboral y viceversa. En el primer caso emergen las palabras "estudié" y "carrera", mientras en el segundo caso aparecen las palabras "cómoda" y "contenta". 


\section{Conclusiones}

La investigación en el área de desajuste educacional analiza los efectos salariales del desajuste entre empleo y área de estudio. Los resultados en esta área indican que entre los egresados de áreas de conocimiento orientadas a la formación de competencias genéricas se observa una mayor probabilidad de desajuste entre empleo y área de estudio respecto de los egresados de áreas de conocimiento orientadas a competencias más específicas. Asimismo, los egresados que trabajan fuera de su área de estudio acceden a salarios menores comparados con egresados empleados en puestos pertinentes. La diferencia en salarios depende del área de estudio y del grado de desajuste entre empleo y área de estudio (Robst, 2007). Es mayor entre egresados de áreas de conocimiento específico respecto de egresados de áreas de conocimiento genéricas, y también es mayor entre quienes reportan trabajar en un empleo completamente no relacionado con su área de estudios versus quienes reportan trabajar en un empleo parcialmente relacionado.

Este estudio analiza la magnitud del desajuste entre empleo y área de estudio y sus efectos en términos de salario y satisfacción con el empleo, para hombres y mujeres, a uno y tres años de haber egresado de la universidad. Entre las mujeres a un año de haber egresado, la mayor probabilidad de desajuste se da entre las egresadas de las áreas de Tecnología y Ciencias Sociales y Humanidades, y entre las empleadas en el sector de Tecnología y Telecomunicaciones y en el sector Comercio. En el caso de los hombres a un año de haber egresado, todos quienes lo hacen del área Ciencias Sociales y Humanidades o trabajan en el sector Construcción se emplean en puestos de trabajo relacionados con su área de estudios. Asimismo, a tres años de haber egresado, el desajuste entre empleo y área de estudio es mayor para las mujeres empleadas en los sectores Financiero e Industrial y para los hombres egresados del área Administración y Comercio. El desajuste es nulo entre los egresados del área Ciencias Sociales y Humanidades.

Las mujeres y hombres que se emplean en puestos no relacionados con su área de estudio obtienen salarios menores que los 
trabajadores que reportan hacerlo en un empleo que sí se relaciona con su área de estudio. El desajuste entre empleo y área de estudios introduce costos salariales adicionales a los ya existentes entre áreas del conocimiento y sectores económicos. Los retornos salariales en el área de Tecnología y en el de Administración y Comercio son estadísticamente iguales entre sí y mayores, a su vez, que los retornos en las áreas de Ciencias Sociales y Humanidades y Arte y Arquitectura, respectivamente. Asimismo, los retornos son mayores en los sectores económicos de Tecnología y Telecomunicaciones, Financiero, Industria, Construcción y Sector Público, comparado con el sector Servicios. Los menores retornos salariales producidos por el desajuste entre empleo y área de estudio se intensifican a tres años de haber egresado.

El análisis de distribuciones de ingreso muestra que el desajuste entre empleo y área de estudio se concentra en tramos de ingreso bajo entre hombres y mujeres a un año de haber egresado y entre las mujeres a tres años de haber egresado. Asimismo, el desajuste se concentra en tramos de ingresos medios en el caso de los hombres a tres años de haber egresado. Esta evidencia induce la existencia de una supuesta endogeneidad entre salario y autopercepción de desajuste, de modo tal que para una determinada profesión, los menores retornos salariales asociados a trabajadores desajustados podrían estar explicados por la mayor autopercepción de desajuste entre quienes obtienen salarios menores a la esperanza promedio de la profesión o por la menor autopercepción de desajuste entre quienes obtienen salarios mayores al promedio esperado en la profesión. Si la percepción de desajuste dependiese de las expectativas salariales en este doble sentido, el modelo de salarios debiera incorporar variables de control relacionadas con las expectativas salariales de los egresados, que a su vez fueran independientes de la percepción de desajuste. Los investigadores que han modelado la formación de expectativas salariales en estudiantes de educación superior en Chile sugieren que el error de predicción de salarios se concentra entre los estudiantes de bajo nivel socioeconómico y bajo rendimiento académico (Hastings, Neilson \& Zimmerman, 2015) quienes, a su vez, enfrentan menores probabilidades de titulación. De esta manera, un modelo de salarios calibrado para una muestra de egresados 
universitarios que incorpora variables de desempeño y eficacia académica y controla las expectativas salariales de los egresados considerando las áreas del conocimiento y el sector económico del empleo actual, es capaz de atenuar los potenciales sesgos atribuibles a la formación de expectativas salariales. Una alternativa al uso de variables subjetivas para medir el desajuste entre empleo y área de estudio es construir una variable que compare el título universitario con el cargo en el empleo actual. Mientras esta variable elimina el factor subjetivo asociado al entrevistado, introduce subjetividad a través del algoritmo utilizado para comparar el ajuste entre áreas de estudio y tipos de empleo (nótese el caso de un egresado del área Salud que ocupa cargos de gestión).

En materia de satisfacción laboral, los resultados señalan que esta depende positivamente del salario, con excepción de los hombres a tres años de haber egresado, donde el salario es sustituido por el desajuste entre empleo y área de estudio como factor relacionado (negativamente) con la satisfacción con el empleo. Mientras el efecto negativo del desajuste entre empleo y área de estudio es transversal entre hombres y mujeres en las distintas etapas de la trayectoria laboral en términos del salario, su efecto sobre la satisfacción se concentra en los hombres en la etapa de progresión laboral, inhibiendo en ese caso el efecto positivo del salario sobre la satisfacción laboral. El análisis semántico refuerza la conclusión de que los efectos no pecuniarios del desajuste entre empleo y área de estudio se expresan en el caso de los hombres en la etapa de progresión laboral, y se reproducen discursivamente como una insatisfacción asociada con una menor expectativa de desarrollo profesional en el empleo actual.

La caracterización de trayectorias educativas indica la existencia de un patrón de progresión académica diferenciado por áreas del conocimiento. Los egresados de las áreas de Tecnología y Arte y Arquitectura demoran en promedio más tiempo en titularse y poseen menores promedios finales de egreso que sus pares egresados de las áreas de Administración y Comercio y Ciencias Sociales y Humanidades. Mientras los retornos salariales son mayores entre egresados de las áreas Tecnología, y Administración y Comercio comparados con los observados en las áreas de Ciencias Sociales y 
Humanidades, y Arte y Arquitectura, la satisfacción laboral es menos frecuente entre egresados del área Tecnología. Los resultados de este estudio indican que el salario tendría un efecto compensatorio frente a la menor satisfacción laboral y el mayor tiempo requerido para alcanzar la titulación entre los egresados del área Tecnología. En contraposición, los costos pecuniarios y no pecuniarios del desajuste entre empleo y área de estudio se concentrarían entre los hombres egresados del área Administración y Comercio, quienes a su vez obtienen salarios similares y enfrentan menores tiempos de titulación que sus pares del área Tecnología.

\section{Referencias}

Becker, G. (1964). Human capital: A theoretical and empirical analysis with special reference to education. Chicago: University of Chicago Press.

Bingley, P., Corak, M., \& Westergard-Nielsen, N. (2011). The intergenerational transmission of employers in Canada and Denmark (IZA Discussion Paper No. 5593). Bonn: Institute for the Study of Labor.

Borgen, N. (2015). College quality and the positive selection hypothesis: The "second filter" on family background in high-paid jobs. Research in Social Stratification and Mobility, 39, 32-47. https://doi.org/10.1016/j. rssm.2014.11.001

Hartog, J. (2000). Over-education and earnings: where are we, where should we go? Economics of Education Review, 19(2), 131-147. https://doi. org/10.1016/s0272-7757(99)00050-3

Hastings, J., Neilson, C., \& Zimmerman, S. (2015). The effects of earnings disclosure on college enrollment decisions (NBER Working Papers Series). Recuperado de https://www.nber.org/papers/w21300

Marginson, S. (2016). Higher education and the common good. Melbourne: Melbourne University Press.

Mautner, G. (2015). Checks and balances: how corpus linguistics can contribute to CDA. En R. Wodak \& M. Meyer (Eds.), Methods of critical discourse studies (pp. 154-179). CA: Sage Publications.

Melguizo, T. \& Wolniak, G. (2012). The earnings benefits of majoring in STEM fields among high-achieving minority students. Research in Higher Education, 53(4), 383-405. https://doi.org/10.1007/s11162011-9238-z 
Meller, P. (2010). Carreras universitarias: rentabilidad, selectividad y discriminación. Santiago de Chile: Uqbar Editores.

Mincer, J. (1958). Investment in human capital and personal income distribution. Journal of Political Economy, 66(4), 281-302. https://doi. org/10.1086/258055

Ministerio de Educación de Chile (2018). Indicadores de empleabilidad e ingresos. Obtenido de http://www.mifuturo.cl/index.php/futurolaboral/buscador-por-carrera-d-institucion\#

Montenegro, C. E. \& Patrinos, H. A. (2014). Comparable estimates of returns to schooling around the world. Recuperado de http://documents.worldbank. org/curated/en/830831468147839247/pdf/WPS7020.pdf

Polachek, S. W. (1978). Sex differences in college major. Industrial and labor relations review, 31(4), 498-508. https://doi. org/10.1177/001979397803100406

Polachek, S. W. (1981). Occupational self-selection: A human capital approach to sex differences in occupational structure. The Review of Economics and Statistics, 63(1), 60-69. https://doi.org/10.2307/1924218

Psacharopoulos, G. (1994). Returns to investment in education: A global update. World Development, 22(9), 1325-1343. https://doi. org/10.1016/0305-750x(94)90007-8

Rivera, L. (2011). Ivies, extracurriculars, and exclusion: Elite employers' use of educational credentials. Research in Social Stratification and Mobility, 29(1), 71-90. https://doi.org/10.1016/j.rssm.2010.12.001

Robst, J. (2007). Education and job match: The relatedness of college major and work. Economics of Education Review, 26(4), 397-407. https://doi. org/10.1016/j.econedurev.2006.08.003

Roksa, J. \& Levey, T. (2010). What can you do with that degree? College major and occupational status of college graduates over time. Social Forces, 89(2), 389-416. https://doi.org/10.1353/sof.2010.0085

Shaw, K. (1984). A formulation of the earnings function using the concept of occupational Investment. The Journal of Human Resources, 19(3), 319-340. https://doi.org/10.2307/145876

Sotomayor, J. (2015). Rentabilidad de la educación superior técnica entregada por los Centros de Formación Técnica (Tesis para optar al grado de Magíster en Políticas Públicas inédita). Universidad de Chile, Santiago, Chile. 
Teichler, U. (1999). Research on the relationships between higher education and the world of work: Past achievements, problems and new challenges. Higher Education, 38(2), 169-190. https://doi. org/10.1163/9789087907563_006

Tholen, G., Brown, P., Power, S., \& Allouch, A. (2013). The role of networks and connections in educational elites' labour market entrance. Research in Social Stratification and Mobility, 34, 142-154. https://doi. org/10.1016/j.rssm.2013.10.003

Urzúa, S. (2012). La rentabilidad de la educación superior en Chile. Estudios Públicos, 125. Recuperado de https://www.cepchile.cl/cep/site/ artic/20160304/asocfile/20160304095900/rev125_SUrzua.pdf

Wolniak, G., Seifert, T., Reed, E., \& Pascarella, E. (2008). College majors and social mobility. Research in Social Stratification and Mobility, 26(2), 123-139. https://doi.org/10.1016/j.rssm.2008.02.002

Recibido: 05/11/2018

Aceptado: 07/06/2019 